\title{
Comparison of HbA1c and FBS among Diabetics and Non-diabetics to Evaluate Role of HbA1c as a Screening Tool
}

\author{
Bachu L ${ }^{1}$, Siddiqui $\mathbf{I A}^{2}$, Neha ${ }^{3}$ \\ Dr. Bachu Laxmikanth ${ }^{1}$, Dr. Imran Ahmed Siddiqui ${ }^{2}, \mathrm{Neha}^{3}$ \\ ${ }^{1}$ Asst Professor, Department of Biochemistry, ShriSathya Sai Medical College \& Research Institute, Chennai. \\ ${ }^{2}$ Specialist, Department of Biochemistry, ESIC Super Specialty Hospital, Hyderabad. ${ }^{3} \mathrm{I}^{\text {st }}$ MBBS Student, ShriSathya Sai \\ Medical College \& Research Institute, Chennai.
}

Address for Correspondence: Dr. B. Laxmikanth, Email: laxmikanth.bachu@gmail.com

\begin{abstract}
Background: The increase in the incidence of Diabetes in both the urban and rural sectors of population demands a proper screening strategy for early diagnosis, to delay the complications associated with this disorder. Aim: To evaluate HbA1c as a diagnostic tool for screening purposes at the community level. Materials and Methods: 50 Type 2 Diabetics were included as cases and 50 healthy individuals were taken as controls in this study. FBS and HbA1c were estimated in them, and the data was statistically analyzed using SPSS software version17. Results: A significantly $(\mathrm{p}<0.001)$ strong and positive correlation between FBS and HbA1c with a "r" value 0.908 was observed. HbA1c showed $100 \%$ sensitivity and specificity at a best cut off value of $6.7 \%$. Conclusion: Hbalc can be used as an effective screening tool at the community level, provided that the test should be performed using a method that is standardized.
\end{abstract}

Key-words: FBS, HbA1c, Type 2 Diabetes

\section{Introduction}

The high prevalence of diabetes mellitus in the recent years has emerged as a worldwide public health problem, with type 2 accounting for $85-90 \%$ of cases. ${ }^{1}$ Diabetes is under diagnosed as the average lag between onset and diagnosis is 7 years. ${ }^{2,3,1,4}$ Early diagnosis, lifestyle modification, and tight glycemic control can reduce the risk of long-term complications. $^{5,3,6}$ Fasting plasma glucose (FPG) and oral glucose tolerance test (OGTT) are the most widely used screening tests for detection of diabetes. Both the tests measure blood glucose. The

Manuscript received: $21^{\text {st }}$ Aug 2013

Reviewed: $23^{\text {rd }}$ Aug 2013

Author Corrected: $26^{\text {th }}$ Aug 2013

Accepted for Publication: $30^{\text {th }}$ Aug 2013 problems with blood glucose estimations include high individual biological variability, preanalytical variability like the method of collection and storage, lifestyle measures like exercise and calorie restriction and difficulty in ensuring fasting state. ${ }^{7}$

The glycated haemoglobin $\left(\mathrm{HbA}_{1 \mathrm{c}}\right)$ test has been suggested as an alternative screening test for Type 2 diabetes. ${ }^{1} \mathrm{HbA} 1 \mathrm{c}$ overcomes many of these difficulties as fasting state is not required, analytical variability is less than $2 \%$ and gives glycemic status over the past $2-3$ months. ${ }^{7,6} \mathrm{HbA} 1 \mathrm{C}$ values are relatively stable after collection, and the recent introduction of a new reference method to calibrate all 
HbA1C assay instruments should further improve HbA1C assay standardization.

There are recommendations to use $\mathrm{HbAlc} \geq 6.5 \%$ as a diagnostic tool to detect type 2 diabetes based on the International Expert Committee (IEC) in 2009, the American Diabetes Association (ADA) in $2010^{10,12}$ and the World Health Organization (WHO) in 2011.

This cut-point represents the approximate level above which prevalent retinopathy begins to increase. ${ }^{8}$ Its recommendation for diagnosis of diabetes mellitus has evoked mixed response worldwide. The diagnostic test should be performed using a method that is certified by the National Glycohemoglobin Standardization Program (NGSP) and standardized or traceable to the Diabetes Control and Complications Trial (DCCT) reference assay. ${ }^{9}$

\section{Materials \& Methods}

In this study subjects were divided into 50 cases and 50 controls. Cases included recently diagnosed Type2 Diabetics $(<1 \mathrm{yr})$ in the age group of 20 to 40 years and controls comprised of healthy individuals not suffering from any ailments in the same age group i.e. $20-40$ years.

\section{Exclusion Criteria:}

- Type 1 diabetics

- Individuals suffering from any condition that changes red cell turnover, such as hemolytic anemia, chronic malaria, major blood loss, glucose-6-phosphate dehydrogenase deficiency, sickle cell anemia or blood transfusions, hemoglobinopathies, recent hemolysis

- Individuals with high triglyceride levels

- Individuals taking drugs like salicylates, vitamin $\mathrm{C}$ and vitamin $\mathrm{E}$

In both these groups FBS and HbA1c were estimated in the blood samples taken from them after taking written consent. After an overnight fast, peripheral venous blood samples were collected in two vaccutainers $5 \mathrm{ml}$ in gel vaccutainer and $2 \mathrm{ml}$ in the EDTA vaccutainer.

Serum separated after centrifuge; was used to analyze FBS by GOD-POD method. The EDTA sample was used to measure $\mathrm{HbA} 1 \mathrm{C}$ that was determined by Ion-exchange resin method. The association between HbA1c and FBS and also their sensitivity, specificity and predictive values in detection of abnormal values of each other were determined using SPSS software version 17.

\section{Results}

Table 1: Mean FBS and HbA1c value

\begin{tabular}{|c|c|c|c|c|}
\hline Parameter & \multicolumn{2}{|c|}{ Mean \pm 2SD } & T value & Significance \\
\hline & Controls & Cases & & \\
\hline FBS & $85.4 \pm 19.86$ & $213.38 \pm 148.2$ & 12.103 & $<0.001$ \\
\hline HbA1c & $5.36 \pm 0.64$ & $9.22 \pm 3.04$ & 17.542 & $<0.001$ \\
\hline
\end{tabular}

Data obtained was analyzed using SPSS v 17 software. It was observed that the mean FBS in control group $(n=50)$ and diabetic group $(\mathrm{n}=50)$ was $85.4 \mathrm{mg} / \mathrm{dl}( \pm 19.86)$ and $213.4 \mathrm{mg} / \mathrm{dl}( \pm 148.2)$ respectively.

The difference in mean was compared using independent sample $t$ test and it was observed to be significantly higher in diabetics than controls $(\mathrm{p}<0.001)$ at a t value of 12.103. Mean HbA1c in control group was $5.36 \pm 0.64$ and in diabetic group was $9.2 \pm$ 3.0 , the mean difference was significantly more in diabetics $(\mathrm{p}=<0.001)$ at a t value 17.54 
Table 2: Cut of value on the basis of ROC curve for sensitivity and specificity

\begin{tabular}{|c|c|c|c|c|}
\hline Parameter & AUC & Best cut off value & Sensitivity & Specificity \\
\hline FBS & 1.000 & 117 & $100 \%$ & $100 \%$ \\
\hline HbA1c & 1.000 & 6.7 & $100 \%$ & $100 \%$ \\
\hline
\end{tabular}

Using ROC curve analysis it was observed that at a best cut off value of $117.0 \mathrm{mg} / \mathrm{dl}$, FBS had a sensitivity and specificity of $100 \%$ respectively in differentiating cases from controls compared to HbAlc which showed a similar 100\% sensitivity and specificity at a best cut off value of $6.7 \%$ and the positive predictive value for both the parameters at above mentioned best cut off value was $100 \%$.

If we consider the best cut of value for Fbs at $103.5 \mathrm{mg} / \mathrm{dl}$ and $\mathrm{HbA} 1 \mathrm{c}$ at $6.05 \%$ we observe a decrease in specificity to $98 \%$ and sensitivity remains $100 \%$, this combination would be more helpful in differentiating the prediabetics or early diabetics from non diabetic population as the negative predictive value was $100 \%$ for the above sensitivity and specificity.

Table 3: Correlation between FBS AND HbA1c

\begin{tabular}{|c|c|c|c|}
\hline & & Fbs & hba1c \\
\hline \multirow[t]{3}{*}{ Fbs } & Pearson Correlation & 1 & $.908^{* *}$ \\
\hline & Sig. (2-tailed) & & .000 \\
\hline & $\mathrm{N}$ & 100 & 100 \\
\hline \multirow[t]{3}{*}{ hbalc } & Pearson Correlation & $.908^{* *}$ & 1 \\
\hline & Sig. (2-tailed) & .000 & \\
\hline & $\mathrm{N}$ & 100 & 100 \\
\hline \multicolumn{4}{|c|}{ **. Correlation is significant at the 0.01 level (2-tailed). } \\
\hline
\end{tabular}

We also observed a significantly $(\mathrm{p}<0.001)$ strong and positive correlation between FBS and HbA1c with a " $r$ " value 0.908 , suggesting increase in FBS will lead to increase in HbAlc.

On subjecting the patient data to ROC curve analysis it was observed that both FBS and HbA1c had an Area under the curve of 1.0 . At the best cut of value $117 \mathrm{mg} / \mathrm{dl}$ and $6.7 \%$ respectively both the parameters were found to be $100 \%$ sensitive and 100 $\%$ specific in differentiating the diabetic patients from non diabetic.

\section{Discussion}

In the present study which was aimed at validating the use of $\mathrm{HbA} 1 \mathrm{c}$ as a screening modality at the community level, it was found that $\mathrm{HbA} 1 \mathrm{c}$ has some advantages over the age old FBS. HbA1c is unaffected by transient hyperglycemia from acute stress or illness ${ }^{3}$. $\mathrm{HbA}_{1 \mathrm{c}}$ is related to both elevated OGTT and FPG, and the various complications, ${ }^{1}$ therefore it can be used for 
assessing the risk of complications of diabetes as well as for monitoring glycemic control.

HbAlc seems a more practical alternative, as it is an established measure of long-term glycemia ${ }^{3,10}$ and also correlates directly with subsequent development and progression of microvascular complications. ${ }^{9}$ Thus it is helpful in early detection of cases in order to prolong the occurrence of complications

It is rare for the screening tests to have both high sensitivity and specificity ${ }^{1}$. In the case of diabetes, which is a relatively common disease, the efficiency of screening, and therefore the specificity of the test used, is arguably more important.

However in the present study HbA1c had $100 \%$ specificity which is a prerequisite for a good screening test. HbAlc value of $6.5 \%$ has a very high specificity and is a useful supportive marker to diagnose diabetes ${ }^{11}$ and as per this study a HbA1c value of $6.7 \%$ has good specificity and thus is in close agreement.

The $\mathrm{HbA}_{1 \mathrm{c}}$ cut-off point of $>6.1 \%$ was the recommended optimum cut-off point for $\mathrm{HbA}_{1 \mathrm{c}}$ in most reviewed studies; however, there is an argument for population-specific cutoff points as optimum cut-offs vary by ethnic group, age, gender and population prevalence of diabetes.

HbA1c laboratory methods are now well standardized and reliable. The errors caused by nonglycemic factors affecting HbAlc such as hemoglobinopathies are infrequent and can be minimized by confirming the diagnosis of diabetes with a plasma glucose (PG)-specific test. $^{2}$

It has been shown that risk stratification improves the predictive validity of $\mathrm{HbA} 1 \mathrm{c}$ in screening for undiagnosed diabetes $^{3}$, this can be applied to the present

study to improve the effectiveness of Hbalc as a screening tool. Also the combined use of FPG and HbA1c levels predicts the progression to diabetes in individuals with no apparent risk $^{12,13,14}$, this is in contrast to the present study which targets the use of $\mathrm{HbAlc}$ as a sole screening test.

According to Ghazanfari $\mathrm{Z}$ et $\mathrm{al}^{5}$ there was a relatively strong association of HbAlc with FBS which is in concordance with this study as it was observed that a significantly $(\mathrm{p}<0.001)$ strong and positive correlation existed between FBS and HbA1c with a "r" value 0.908, suggesting increase in FBS will lead to increase in HbA1c.

\section{Funding: Nil}

Conflict of interest: Nil

Permission from IRB: Yes

\section{Conclusion}

Although screening with HbAlc would improve detection of undiagnosed diabetes, standardization of the procedure used and cost-effectiveness studies are needed before implementation of specific screening strategies using HbA1c.

\section{References}

1) Bennett CM, Guo M, Dharmage SC. HbA1c as a screening tool for detection of Type 2 diabetes: a 
systematic review. Diabetic Medicine. April 2007; 24(4):333-343.

2) Saudek CD, Herman WH, Sacks DB, Bergenstal RM, Edelman D, Davidson MB. A new look at screening and diagnosing diabetes mellitus. J Clin Endocrinol Metab. July 2008; 93(7):2447-53.

3) Ginde AA, Cagliero E, Nathan DM, Camargo CA Jr. Value of risk stratification to increase the predictive validity of $\mathrm{HbA} 1 \mathrm{c}$ in screening for undiagnosed diabetes in the US population. J Gen Intern Med. Sep 2008; 23(9):1346-53.

4) Deborah J. Wexler, David M. Nathan, Richard W. Grant, Susan Regan, Allison L. Van Leuvan and Enrico Cagliero. Prevalence of Elevated Hemoglobin A1c among Patients Admitted to the Hospital without a Diagnosis of Diabetes. J Clin Endocrinol Metab. 2008; 93:4238- 4244.

5) Ghazanfari Z, Haghdoost AA, Alizadeh SM, Atapour J, Zolala F. A Comparison of HbA1c and Fasting Blood Sugar Tests in General Population. Int J Prev Med. 2010 ;1(3):187-94

6) Silverman, Pahk RA, Carbone R, Wells M, Mitzner E, Burris R, Kelson K, Grella JR, Katzeff R, Harvey. The Relationship of Plasma Glucose and HbA1c Levels among Emergency Department Patients with No Prior History of Diabetes Mellitus. Acamedic Emergency Medicine. 2006; $13: 722-726$.

7) Kumar PR, Bhansali A, Ravikiran M, Bhansali S, Dutta P, Thakur JS, Sachdeva N, Bhadada SK, Walia R. . Utility of Glycated Hemoglobin in Diagnosing Type 2 Diabetes
Mellitus: A Community-Based Study. J Clin Endocrinol Metab. 2010; 95: 2832-2835.

8) SA Mostafa, K Khunti, ES Kilpatrick, D Webb, BT Srinivasan, LJ Gray and MJ Davies. Diagnostic performance of using one- or two-HbAlc cut-point strategies to detect undiagnosed type 2 diabetes and impaired glucose regulation within a multi-ethnic population. Diabetes and Vascular Disease Research. Jan 2013;10(1):84-92.

9) Mahajan RD , Mishra B. Using Glycated Hemoglobin HbA1c for diagnosis of Diabetes mellitus: An Indian perspective. Int J Biol Med Res. 2011; 2(2): 508-512.

10) Pradhan $A D$, Rifai N, Buring JE, Ridker PM. HbA1c Predicts Diabetes but not Cardiovascular Disease in NonDiabetic Women. The American Journal Of Medicine. August 2007; 120(8):720-727.

11) Tanaka Y, Atsumi Y, Matsuoka K, Mokubo A, Asahina T, Hosokawa K, Shimada S, Matsunaga H, Takagi M, Ogawa O, Onuma T, Kawamori R. Usefulness of stable $\mathrm{HbA}(1 \mathrm{c})$ for supportive marker to diagnose diabetes mellitus in Japanese subjects. Diabetes Res Clin Pract. Jul 2001;53(1):41-5.

12) Inoue $\mathrm{K}$, Matsumoto $\mathrm{M}$, Kobayashi $\mathrm{Y}$. The combination of fasting plasma glucose and glycosylated hemoglobin predicts type 2 diabetes in Japanese workers. Diabetes Res Clin Pract. Sep 2007;77(3):451-8.

13) E. Mannucci, A. Ognibene, I. Sposato, M. Brogi, G. Gallori. Fasting plasma glucose and glycated haemoglobin in the screening of diabetes and impaired glucose tolerance. 
ACTA DIABETOLOGICA, December 2003; 40(4): 181186.

14) Norberg M, Eriksson JW, Lindahl B, Andersson

C, Rolandsson O, Stenlund H, Weinehall L A combination of HbAlc, fasting glucose and BMI is effective in screening for individuals at risk of future type 2 diabetes: OGTT is not needed. J Intern Med. Sep 2006;260(3):26371.

\section{How to cite this article?}

Bachu L, Siddiqui IA, Neha. Comparison of HbA1c and FBS among Diabetics and Non-diabetics to evaluate Role of HbA1c as a Screening Tool. Int J Med Res Rev 2013;1(3):125-130. doi: 10.17511/ijmrr.2013.i03.08 